\title{
Proteomic analysis defines kinase taxonomies specific for subtypes of breast cancer
}

\author{
Kyla A.L. Collins ${ }^{1, *}$, Timothy J. Stuhlmiller ${ }^{2,3, *}$, Jon S. Zawistowski ${ }^{2,3}$, Michael P. \\ East $^{2,3}$, Trang T. Pham ${ }^{2,3}$, Claire R. Hall ${ }^{4}$, Daniel R. Goulet 2,3 , Samantha M. Bevill2,3, \\ Steven P. Angus ${ }^{2,3}$, Sara H. Velarde ${ }^{2,3}$, Noah Sciaky ${ }^{2}$, Tudor I. Oprea ${ }^{5,6}$, Lee M. \\ Graves $^{2,3}$, Gary L. Johnson ${ }^{2,3}$ and Shawn M. Gomez ${ }^{1,2,4}$ \\ ${ }^{1}$ Curriculum in Bioinformatics and Computational Biology, University of North Carolina at Chapel Hill, Chapel Hill, NC 27514, USA \\ ${ }^{2}$ Department of Pharmacology, University of North Carolina at Chapel Hill, Chapel Hill, NC 27514, USA \\ ${ }^{3}$ Lineberger Comprehensive Cancer Center, University of North Carolina at Chapel Hill, Chapel Hill, NC 27514, USA \\ ${ }^{4}$ Joint Department of Biomedical Engineering, University of North Carolina at Chapel Hill and North Carolina State University, \\ Chapel Hill, NC 27514, USA \\ ${ }^{5}$ Translational Informatics Division, School of Medicine, University of New Mexico, Albuquerque, NM 87106, USA \\ ${ }^{6}$ UNM Comprehensive Cancer Center, University of New Mexico, Albuquerque, NM 87131, USA \\ *These authors contributed equally to this work \\ Correspondence to: Gary L. Johnson, email: gary_johnson@med.unc.edu \\ Shawn M. Gomez, email: smgomez@unc.edu \\ Keywords: cancer biology; proteomics; kinase signaling \\ Received: September 27, 2017 Accepted: January 19, 2018 Epub: January 29, 2018 Published: March 20, 2018 \\ Copyright: Collins et al. This is an open-access article distributed under the terms of the Creative Commons Attribution License 3.0 \\ (CC BY 3.0), which permits unrestricted use, distribution, and reproduction in any medium, provided the original author and source \\ are credited.
}

\section{ABSTRACT}

Multiplexed small molecule inhibitors covalently bound to Sepharose beads (MIBs) were used to capture functional kinases in luminal, HER2-enriched and triple negative (basal-like and claudin-low) breast cancer cell lines and tumors. Kinase MIB-binding profiles at baseline without perturbation proteomically distinguished the four breast cancer subtypes. Understudied kinases, whose disease associations and pharmacology are generally unexplored, were highly represented in MIB-binding taxonomies and are integrated into signaling subnetworks with kinases that have been previously well characterized in breast cancer. Computationally it was possible to define subtypes using profiles of less than $\mathbf{5 0}$ of the more than $\mathbf{3 0 0}$ kinases bound to MIBs that included understudied as well as metabolic and lipid kinases. Furthermore, analysis of MIB-binding profiles established potential functional annotations for these understudied kinases. Thus, comprehensive MIBs-based capture of kinases provides a unique proteomics-based method for integration of poorly characterized kinases of the understudied kinome into functional subnetworks in breast cancer cells and tumors that is not possible using genomic strategies. The MIB-binding profiles readily defined subtype-selective differential adaptive kinome reprogramming in response to targeted kinase inhibition, demonstrating how MIB profiles can be used in determining dynamic kinome changes that result in subtype selective phenotypic state changes.

\section{INTRODUCTION}

In 2014 the NIH established an initiative entitled Illuminating the Druggable Genome (IDG) to determine the function of understudied proteins including kinases encoded in the human genome (https://commonfund. nih.gov/idg/index). The human kinome is comprised of $\sim 520$ protein kinases that are highly druggable using both competitive small molecule and allosteric inhibitors. Including both lipid and metabolic kinases enlarges this family to $\sim 634$ (druggable) kinases. Of the protein kinases, the function of about one-third are poorly defined with 
the function and regulation of 50-100 kinases remaining largely unknown. To categorize our understanding of proteins in the human genome the IDG Knowledge Management Center (KMC) (http://targetcentral.ws/index) has developed a set of criteria for target development level (TDL) for druggable proteins such as kinases, G proteincoupled receptors and ion channels [1]. This knowledge base was used to categorize the 634 kinases in the human genome as Tclin (50 kinases), Tchem (390 kinases), Tbio (163 kinases) and Tdark (31 kinases). Using TDL criteria, the KMC defines Tclin as bona fide diseaseinvolved kinases that are drug targets for at least one FDA approved pharmaceutical entity [2]. Tchem includes target kinases having characterized small molecules that bind with high potency (activity $\leq 30 \mathrm{nM}$ ), have active pharmacologic studies in relation to a disease, and likely to have medicinal chemistry efforts seeking highly selective molecules that perturb the functions of the kinase. Tbio is a biology- and disease-focused category that basically includes kinases not included in Tclin or Tchem, but above Tdark criteria. They often have an association with human disease and even small molecules that are less potent (e.g., above $30 \mathrm{nM}$ ). Finally, Tdark includes kinases having the least understood function (e.g., 5 or less publications) and molecular probes for their study are scarce. Tdark kinases are generally poorly characterized for their integration into kinase signaling networks, represent unknowns in disease associations, and are unexplored as drug targets alone or in combination with other proteins. Even with the growing databases of genomic information for different cancers, it is often still unclear how molecular taxonomies translate to phenotype. Additional methods characterizing proteomic taxonomies are needed to understand signaling networks, particularly of protein kinases due to their high druggability potential. Important for this analysis of the cancer kinome is a characterization of understudied kinases (including Tbio and Tdark), which represent a third of the kinome and lack essential functional characterization as well as molecular tools for their manipulation and study [3]. These understudied kinases need to be functionally integrated into kinase networks for a global understanding of kinome dynamics to be achieved both at baseline and in response to perturbation.

We focused on exploring the integration of understudied kinases into kinase networks within the context of breast cancer, which has three primary subtypes that include luminal (further sub-divided into luminal $\mathrm{A}$ and B subtypes) as well as the majority of HER2+ breast cancers along with triple negative breast cancer (TNBC), that can itself be broken into basal-like and claudinlow subtypes [4]. Interestingly, basal-like breast cancer using molecular taxonomies is as different from luminal and HER2+ breast cancers as lung cancer, leading to the proposal that basal-like breast cancers are in fact a unique disease [5, 6]. Estrogen and progesterone receptor dependence and HER2 addiction define vulnerabilities in
luminal/HER2+ breast cancers. However, in basal-like and claudin-low triple negative breast cancer, there are no oncogenic drivers that define a common vulnerability that can be therapeutically targeted.

In an attempt at having a more complete understanding of the integrated kinome in breast cancer, we have developed methods using multiplexed inhibitor beads (MIBs) coupled with mass spectrometry (MIB/MS) that have the ability to bind and identify a large percentage of kinases in the human kinome [7, 8]. By RNA-seq, most cell lines express $\sim 350$ kinases and our MIB-binding profiling captures a significant percentage of the expressed kinome [9]. In the current study, we have compiled the baseline kinase MIB-binding profile using MIB/MS for 15 cell lines representing all four breast cancer subtypes in addition to patient tumors. It was possible to define kinase taxonomies for breast cancers using feature selection methodologies based on the MIB/MS profile of 50 kinases among the kinases captured by MIB/MS that includes understudied protein kinases, lipid and metabolic kinases. Using the baseline MIB-binding state in a machine-learning framework further allowed the classification of breast cancer subtype in primary tumors. Kinases identified within these distinguishing profiles are distributed throughout subfamilies of the kinome, representing multiple subnetworks with a significant representation of understudied kinases. In particular, we utilize a regression approach to integrate known interaction and phosphorylation data with MIB-binding behavior to establish functional subnetworks and associated annotation for 89 understudied kinases, including 22 kinases defined as Tbio or Tdark. These findings demonstrate that determining the functional kinome based on MIB-binding has prognostic value in defining the integration of signaling networks that is not currently possible using genomic strategies.

\section{RESULTS}

\section{Multiplexed kinase inhibitor beads capture kinases from every subfamily and provide a means to assay understudied kinases}

Multiplexed kinase inhibitor beads (MIBs) are a set of Sepharose beads each with a specific covalentlyattached kinase inhibitor [7, 10]. Coupling MIB gravityflow affinity chromatography with mass spectrometry (MIB/MS) provides the ability to capture and identify kinases from whole cell lysates on a kinome scale. Binding of kinases is dependent on the functional expression and activity of the kinase and affinity for the different immobilized inhibitors. To determine the inhibitor bead selective distribution of bound kinases, we assayed kinase capture by six different inhibitors individually covalently coupled to Sepharose beads [8, 10]: CTx-0294885, VI16832, PP58, Purvalanol B, and two custom synthesized molecules, UNC-8088A and UNC-2147A. Four cell lines representative of breast cancer subtypes: HCC1806 (basal-like), SUM159 (claudin-low), MCF7 (luminal), and 
SKBR3 (HER2-enriched) were used for analysis of kinase capture by each bead (Figure 1A). Of these, CTx-0294885 (CTx) and VI-16832 (VI) captured the most total kinases (265 and 254, respectively) and the most unique kinases (32 and 29, respectively). The other four beads bound a lesser number of kinases (PP58, 194 kinases; Purvalanol B, 164; UNC-8088A, 162; UNC-2147A, 130, Figure 1B, Supplementary Table 1). Although UNC-8088A binds the fewest unique kinases (only five), these include the atypical bromodomain and extraterminal (BET) domaincontaining family of chromatin readers BRD2, -3 , and -4 [11]. Hierarchical clustering of identified kinase peptides shows each bead binds a unique set of kinases and UNC-2147A displays the most distinct binding profile selectively enriching the AGC kinases (Figure 1C).

Understudied kinases [3] make up approximately $40 \%$ of the overall expressed kinome and are similarly represented through MIB-binding, with 23-34\% of all kinases captured for any individual bead (Figure 1D; Supplementary Table 2). Characteristics of understudied kinases include: i) integration of the protein kinase in signaling networks is poorly defined, ii) function and/or regulation is poorly defined, iii) activation loop phosphoantibodies and/or IHC grade antibodies may not exist, iv) lack of selective chemical tools for use in characterization of function (e.g., small molecule inhibitors), v) RNAi and CRISPR/Cas9 for knockout/altered expression and cDNAs for overexpression may be primary tools, vi) kinase knockout or altered expression may not provide readily assayable phenotypes (e.g., growth, migration, apoptosis or in vivo function in mouse organ physiology).

Across all MIB/MS runs, 381 kinases in total were identified. Of these, 35 are metabolic and lipid kinases, 346 are protein kinases of which 142 can be considered understudied $(41 \%$ of protein kinases identified) (Figure 1E). The overall distribution of kinases bound indicates CTx and VI are clearly pan-kinase inhibitors (Figure 1F, circle size proportional to number of unique peptides identified per kinase). Purvalanol B also binds kinases across families but to a lesser extent. PP58 has some preference for tyrosine kinases (TKs), and UNC-8088A has preference for TKs, CMGC, and atypical kinases over other families. UNC-2147A, designed for interaction with the binding pocket of AKT, has a strong affinity for AGC kinases lacking in most of the other five kinase inhibitors. CTx, VI, and PP58 have a strong affinity for PRKDC (DNA-PK) not seen with the other three inhibitors. All inhibitor beads display high affinity for many understudied kinases (green circles and text). The most-highly captured understudied kinases across the four cell lines were GAK, SLK, MRCKB, AAK1, TBK1, and NEK9.

Kinases known to be oncogenic drivers in general and/or nodal signaling kinases display anticipated MIBbinding profiles across the different breast cancer subtype cell lines (Figure 1G). For example, SKBR3 (luminal HER2+) and MCF7 (luminal) cells have abundant AKT1/2
MIB-binding. Other well characterized kinases are highly represented in a specific cell line, such as EGFR and FAK1 in HCC1806, EPHA2 and UFO (AXL) in SUM159, IGF1R and KS6B1 (p70 S6K) in MCF7, and HER2/ERBB2 and TGFBR1 in SKBR3 cells. Several understudied kinases also show high selectivity in functional MIBbinding including CDK13, DMPK, SIK3 and TESK1 in MCF7 and CLK4, CDK14 and NLK in SUM159 cells (Figure 1H). Figure 1I and Supplementary Table 1 show kinases whose MIB-binding is greatest in each of the four cell lines, proportional to the number of unique peptides identified. Unsupervised hierarchical clustering illustrates the differences in MIB-binding throughout the kinome for each cell line (Figure 1J). These findings indicate the four cell lines display a unique MIB/MS binding profile for both well characterized and understudied kinases.

\section{Integrating understudied and well characterized kinases by kinome proteomic profiling defines breast cancer subtypes}

We characterized the baseline kinome of 15 breast cancer cell lines representing the four major breast cancer subtypes as previously defined by gene expression profiles [4]. Cell lysates were passed over an affinity column composed of the six kinase inhibitor beads and processed with LC-MS/MS (Figure 2A). Using label-free peptide quantitation measurements, a total of 360 kinases were identified as having at least 3 unique peptides (Supplementary Data File 1). MIB-binding profiles generated from each of the respective cell lines were averaged into a single representative profile and a Pearson correlation matrix between each of these profiles was then generated and hierarchically clustered with a Euclidean distance function. As can be observed in Figure 2B, claudin-low and basal-like cells (TNBC) are readily distinguished from HER2-enriched/luminal cells by MIB profiling of their cellular kinomes. The basal-like HER2amplified cell line HCC1954 clusters with basal-like lines through similarity of kinome profiles and is thus separated from the luminal HER2+ lines. Interestingly, the SKBR3 HER2-enriched cell line shows an intermediate clustering between HCC1954 and other HER2+/luminal cell lines, and a previous report demonstrated SKBR3 patterns as basal-like in functional RNAi screens [12]. Hierarchical clustering of kinases further separated cell lines with the claudin-low phenotype, SUM159, MDA-MB-231 and MDA-MB-468 (basal-like), showing the greatest difference from other cell lines (Figure 2C). SUM229 cells have two subpopulations, a basal-like EpCAM positive/E-cadherin positive (SUM229pos) and a claudinlow EpCAM negative/E-cadherin negative population (SUM229neg). The two populations are genomically similar by exome sequencing, but differ epigenetically [13] and cluster together based on their kinome MIBbinding profile (Figure 2C). 

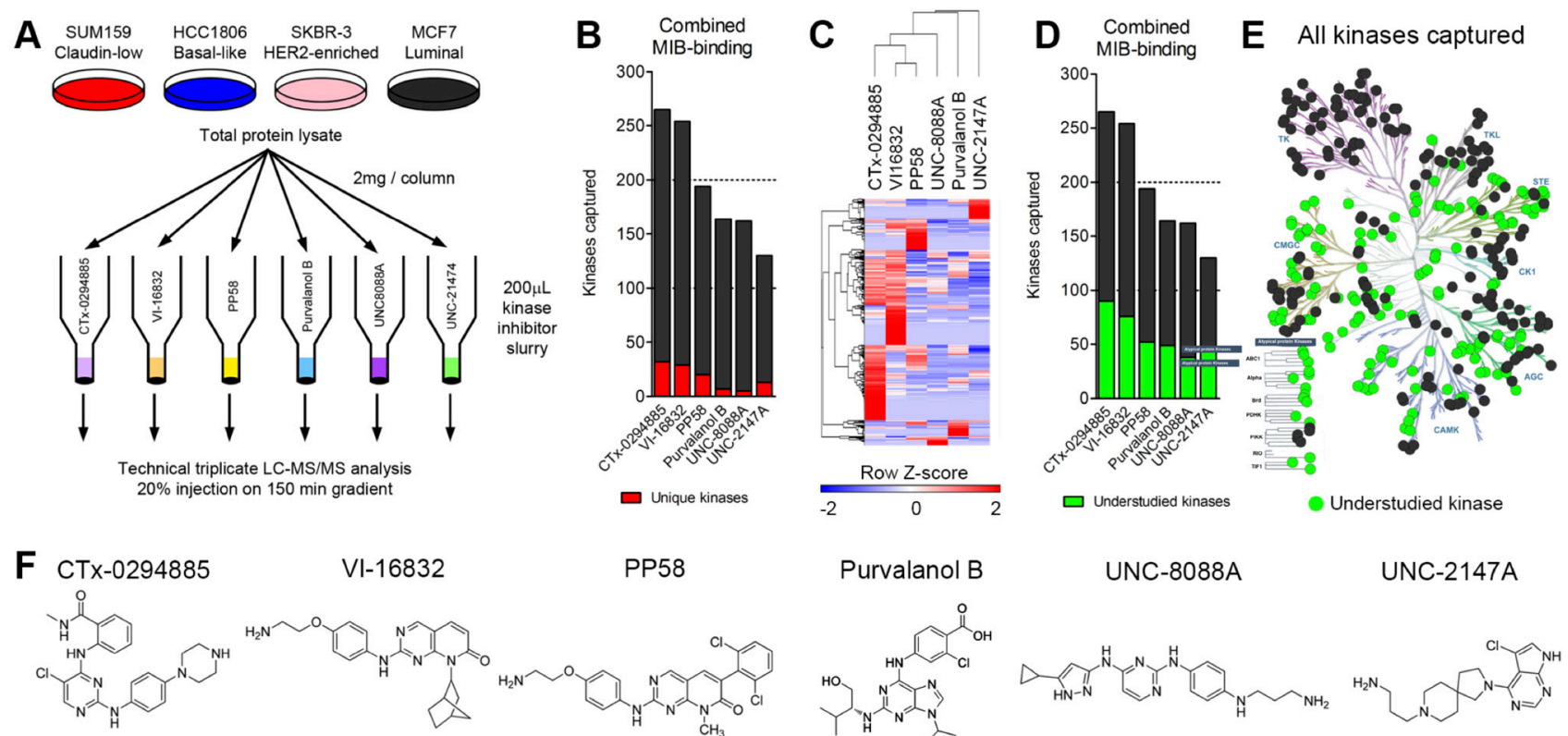

\section{UNC-8088A \\ UNC-2147A}

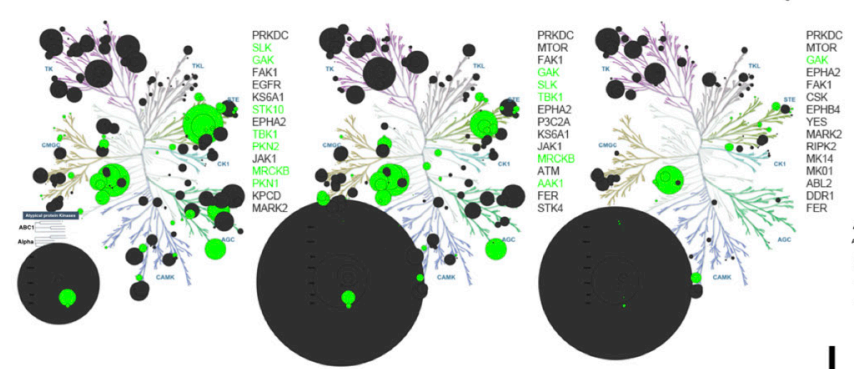

$\mathbf{G}_{\text {MIB-binding of Characterized Kinases }}$
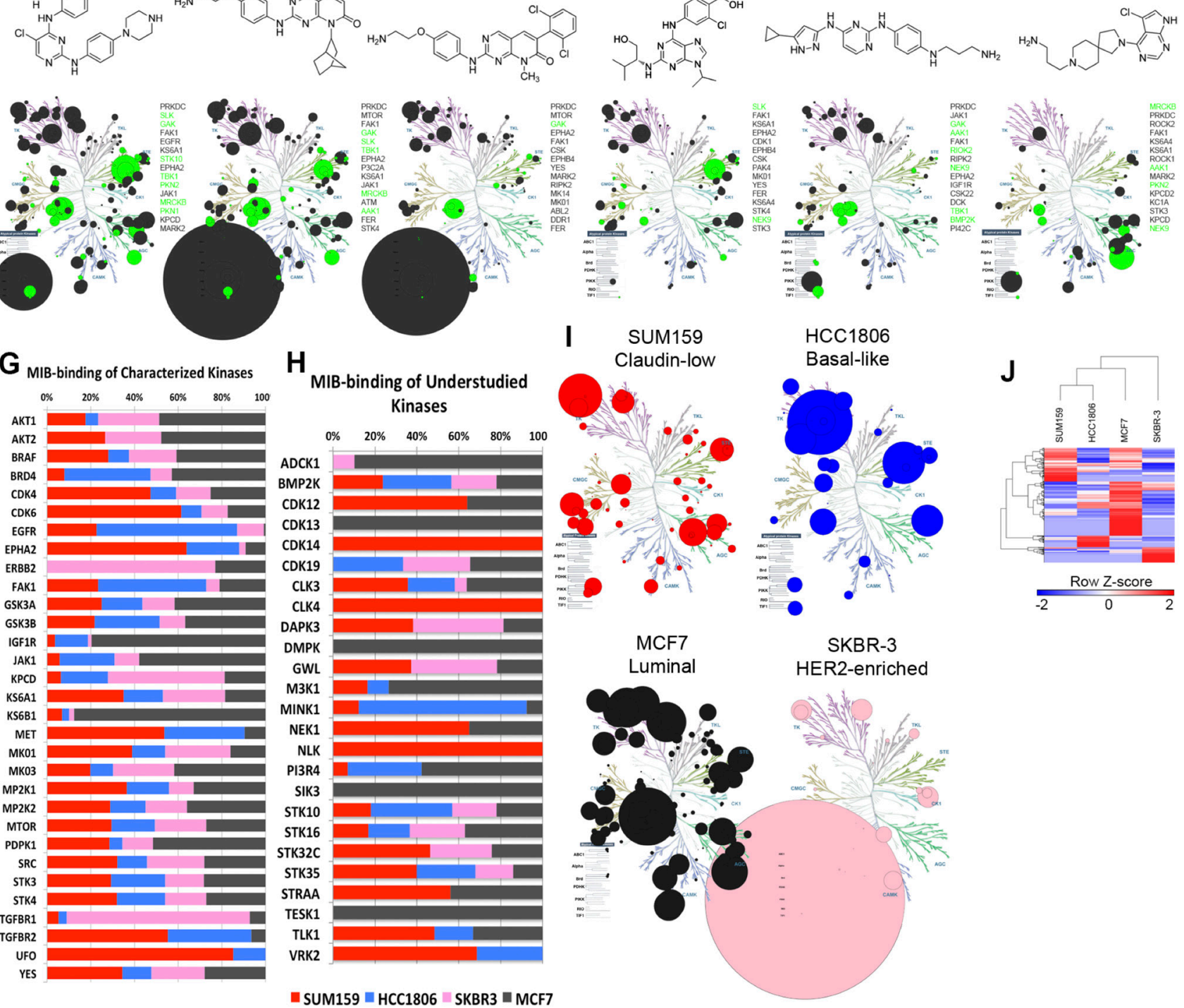

Figure 1: Assessment of multiplexed kinase inhibitor beads (MIBs) for kinase capture across breast cancer subtypes. (A) Experimental design to assess performance of six kinase inhibitor beads. (B) Combined data from all four cell lines assayed shows CTx-0294885 binds the most number of kinases. Number of kinases captured uniquely by each bead is shown in red. (C) Euclidean hierarchical clustering kinase peptides bound by the six beads shows each bead enriches for a distinct set of kinases. UNC-2147A displays the most unique binding profile. (D) A large proportion of kinases captured by MIBs (23-24\%) are understudied or poorly characterized 
(green). (E) 381 kinases were identified across all four cell lines, including 346 protein kinases and 35 metabolic kinases. Of these protein kinases, 142 are understudied (green). (F) Chemical structures and kinase-binding of each inhibitor bead across the kinome. Circle size is proportional to the number of unique peptides identified per kinase. PRKDC (DNA-PK) is over-represented in VI-16832 and PP58 (large circle under Atypical protein kinases). Most beads capture kinases across families but UNC-2147 preferentially enriches for AGC family kinases. Shown to the right of each kinome tree are the 15 most-highly captured kinases for each bead. Green circles and text signify understudied kinases. (G) Comparison of relative binding of characterized kinases across breast cancer cell lines/subtypes. (H) Comparison of relative binding of understudied kinases across breast cancer cell lines/subtypes. (I) Each cell line representing the different breast cancer subtypes displays a unique kinome profile. Only kinases with the greatest number of peptides identified in each cell line are shown. Circle size is proportional to the number of peptides identified. (J) Hierarchical clustering of peptides identified for each kinase (rows) across the cell lines (columns) cluster triple-negative cell lines (SUM159, HCC1806) together and indicates HER2-positive SKBR3 cells have the most distinct kinome profile.

Principal components analysis (PCA) of baseline MIB-binding kinase profiles revealed significant differences between subtypes within the first principal component, clearly separating triple-negative from HER2-enriched and luminal cell lines (Figure 2D). Further separation of the triple-negative group into claudin-low and basal-like subtypes is also readily achieved. Appreciable separation of HER2-enriched cell lines from luminal cell lines is observed in the second principal component, as is that of the basal-like/HER2-amplified cell lines from the basallike and claudin-low lines. A loadings plot, which defines relationships between MIB-binding for each kinase, highlights those kinases with significant variation within subtypes, with numerous understudied kinases being apparent (Figure 2E). Examples of understudied kinases with differences in MIB-binding among cell lines include ADCK1, PKN3, STK17A and TESK1. Similarly, well characterized kinases known for their relevance in breast cancer are observed, such as ERBB2, EPHA1, MET and TGFBR2.

Supervised differential expression analysis of MIBcaptured kinases from claudin-low/basal-like (TNBC) versus HER2/luminal cell lines defined several statistically significant differences (Figure 2F). Multiple Ephrin receptors (EPHA2/A7/B2) and members of the TGF-beta superfamily (TGFBR2, ACVR1) are among the kinases most associated with TNBC while ERBB3 and RET are over-represented in HER2+ and luminal cell lines. Many understudied kinases display higher MIB-binding in HER2+/luminal cells, including DMPK, ADCK1, and TESK1. Individual plots for selected kinases, both characterized and understudied, are shown in Figure 2G, showing distinctive patterns of MIBbinding across subtypes.

\section{Kinase MIB-binding activity is independent from mRNA expression level}

Our results clearly demonstrate that kinase MIBbinding displays strong variation across breast cancer subtypes. Global gene expression measurements have similarly shown subtype-specific dynamics, with expression of selected gene sets being utilized in subtype determination and diagnosis [14-16]. We compared baseline RNAseq measurements with corresponding MIB-capture of protein kinases to assess the relationship between transcript abundance and functional kinome behavior. Similarity of kinase profiles for a given measurement modality was highly similar, with unsupervised hierarchical clustering grouping RNA-seq profiles separately from those derived from MIB-binding (Figure 3A). Furthermore, similarity within a modality was very high, such that breast cancer subtypes were largely clustered correctly, especially when looking at MIB-binding profiles that clearly grouped along luminal, HER2+ and triple negative subtypes.

While within-group correlations were high, normalized RNA-seq was found to have a very poor correspondence to MIB-binding using label-free quantification of kinase peptide abundance. Quantitative comparison for each subtype achieved correlation coefficients of no more than 0.25 , implying that less than $7 \%$ of the observed variation between MIB-binding and RNA abundance in breast cancer subtypes is explained through this relationship. The distribution of the Pearson correlation coefficients of all kinases across all 15 cell lines similarly shows a low correspondence between MIB-binding and RNA-seq (Figure 3B), with the mean correlation being 0.2 . These results are consistent with other work that found the average correlation between gene expression and protein abundance in TCGA colorectal cancer samples to be approximately 0.47 , with a lesser correlation of 0.23 when comparing gene expression and protein variation [17]. A more recent comprehensive analysis of several data sets has further shown that mRNA levels are not predictive of protein levels for a given gene [18]. The low correlation between RNA-seq and MIB-binding suggests that the use of MIB/MS provides a picture of kinome behavior complementary to that provided through RNA expression measures. In particular, these results support the potentially significant role of post-translational and post-transcriptional regulation in kinome dynamics [18-20].

While there is significant overlap, there are a number of kinases that are only observed with one of the applied methods, MIB/MS or RNA-seq (Figure 3C). This discrepancy is partly due to the 50+ RSEM read threshold used here as a positive identification in RNAseq, potentially missing very lowly expressed kinases. Similarly, kinases not observed with MIB/MS but 
identified in RNA-seq may be missed due to being in an inactive, nonfunctional state and/or failure of chosen inhibitors to bind these kinases with adequate affinity. Pseudokinases that do not bind ATP will generally not be captured by MIBs.
While the degree of correlation between RNAseq and MIB-binding can vary significantly for a given kinase, we do observe a broad range of behaviors across cell lines, with representative high and low correlation profiles shown in Figure 3D. ERBB2 is strongly expressed
A

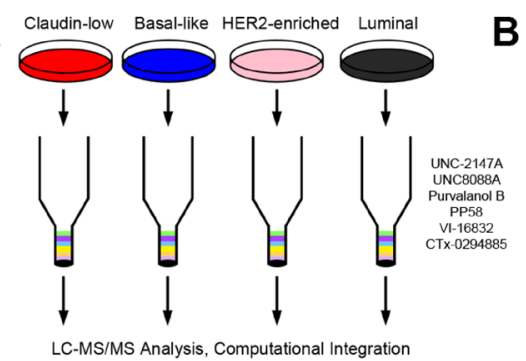

B
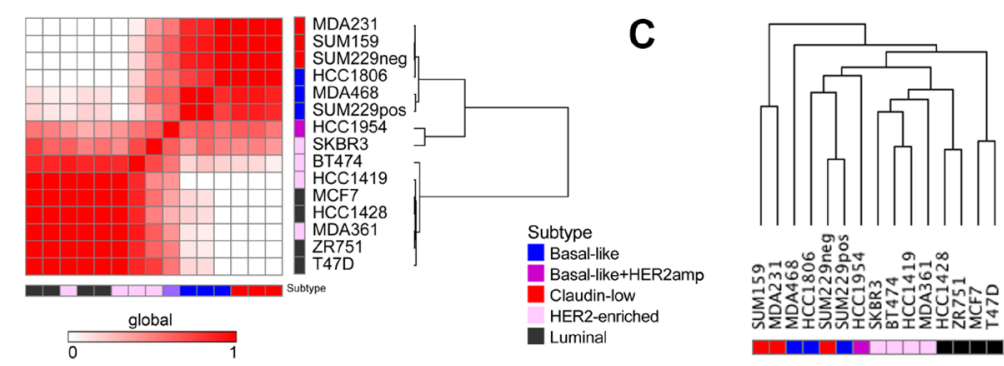

D

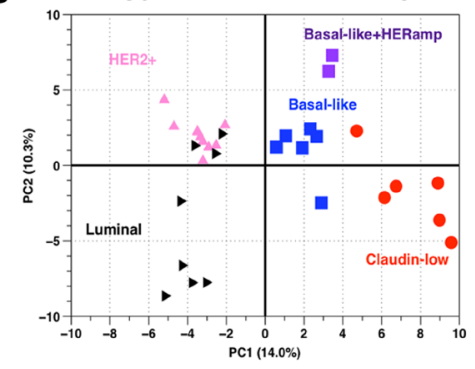

$\mathbf{F}$
E

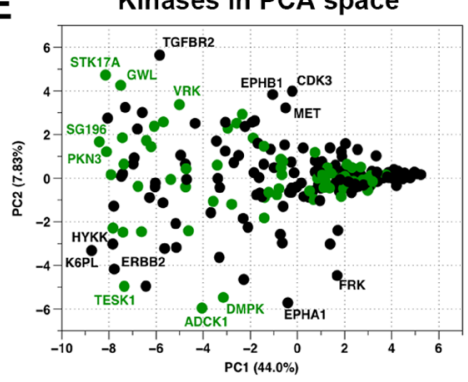

G

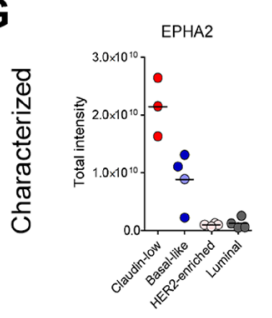

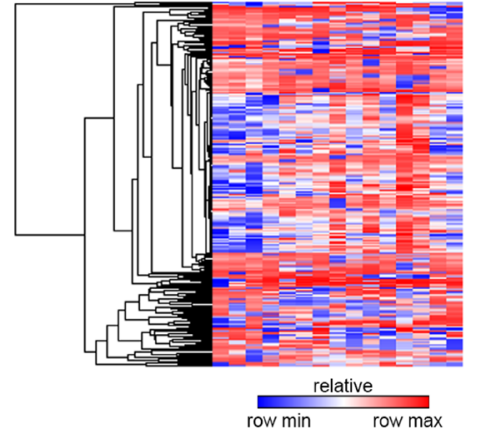

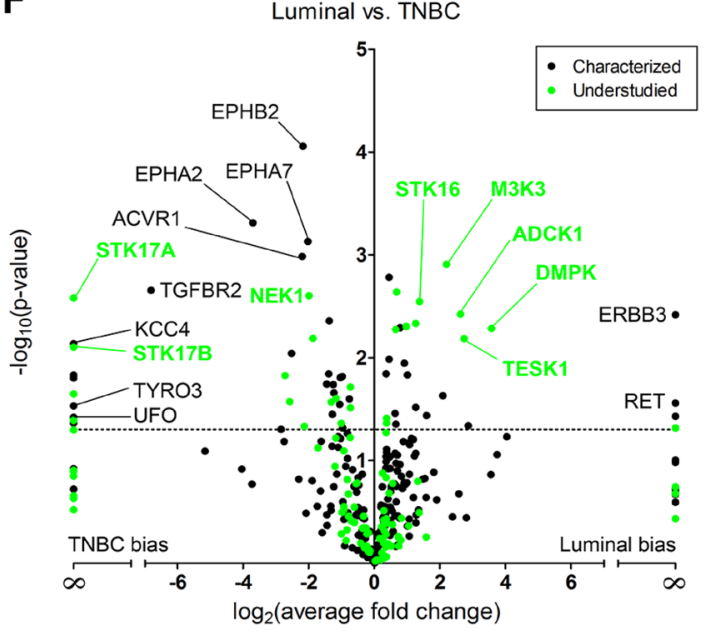

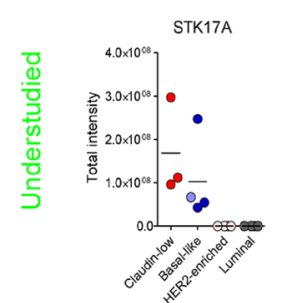
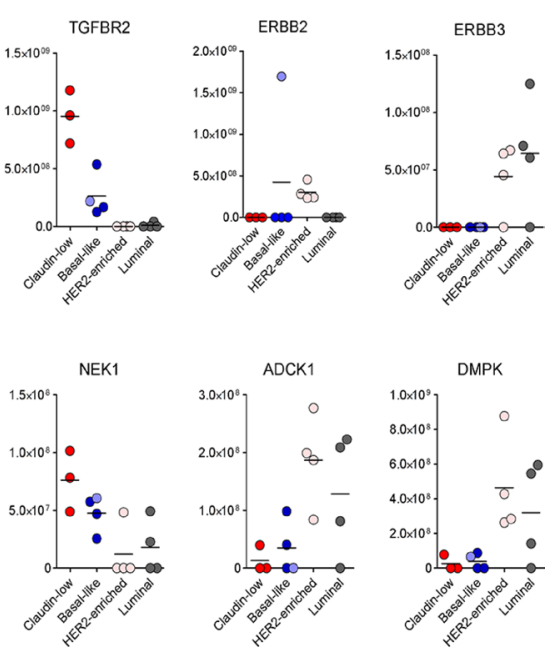
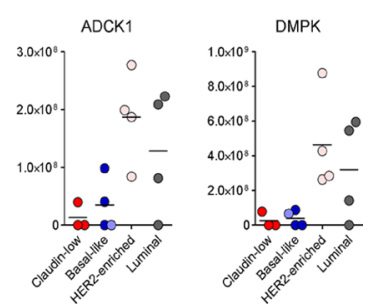

Figure 2: MIB/MS kinome profiling assigns breast cancer cell lines to functional subtypes. (A) Individual samples run across 6-bead composition with LC-MS/MS analysis. (B) Heat map of correlation between MIB/MS samples for cell lines analyzed. Color bars indicated the subtype of each cell line (blue: basal-like, red: claudin-low, pink: HER2-enriched, black: luminal, purple: basal-like/ HER2amp). White in the heat map indicates a low correlation between samples, while red shows higher correlation. Rows and columns are hierarchically clustered. (C) Heat map of MIB/MS average for each of the 15 cell lines analyzed. Rows are kinases; columns are MIB/ MS cell line averages. Color bar for columns indicates the subtype associated to each cell line. Each column is an average of 2 or 3 MIB/ MS samples, depending on the cell line. Colors in the heat map are relative by row minimum (blue) and maximum (red). A total of 254 kinases passed filtering (see Methods). Rows and columns are hierarchically clustered using Euclidean distance and average linkage. (D) Principal Component Analysis (PCA) on the entire MIB/MS data set. PC1 and PC2 account for 14.0\% and 10.3\% of the variance in the data set, respectively. A total of 32 samples across the four subtypes are represented by their subtype (red circle: claudin-low, blue square: basal-like, pink upward triangle: HER2-enriched, black right-pointing triangle: luminal, purple square: basal-like/HER2amp). (E) PCA on the MIB/MS data set to show highly variable kinases across the samples. Characterized and understudied kinases are shown in black and green, respectively. PC1 and PC2 account for $44.0 \%$ and $7.83 \%$ of the variance in the data set, respectively. (F) Volcano plot showing characterized (black) and understudied (green) kinases that are significantly $(p<0.05)$ different between the Luminal/HER2-enriched and TNBC (basal-like/claudin-low) cell line samples in the MIB/MS data set. (G) Profiles of selected characterized (top row) and understudied (bottom row) kinases across breast cancer subtypes. 
in HER2+ cell lines and MIB-binding is correspondingly strong in these samples, consistent with the importance of transcriptional regulation in this kinase's functional output. PDPK1 similarly shows high correlation of expression and MIB-binding across all cell lines, while conversely EPHA1 and M3KL4 (MAP3K21) show very poor correlation between these two data types.

Such proteomic behavioral properties cannot be detected by RNA-seq alone. Together, MIB/MS coupled with RNA-seq provides an integrated perspective, providing a post-transcriptional measure of kinase protein levels. While the functional consequences of posttranscriptional regulation in relation to kinase networks and signaling is not understood, the observed differential relationship of transcript versus protein expression for a subset of poorly correlated kinases suggests an unknown control mechanism possibly involving differential covalent regulatory modifications and/or protein stability. Importantly, MIB/MS measurements avoid the use of recombinant kinases, often used to profile on-target/ off-target inhibitor profiles that are not representative of endogenous kinase complexes [21, 22]. Using MIB capture and MS quantification of endogenous kinases in cell lysates that have associated regulatory subunits and post-translational modifications thus provides a functional measure of cellular kinase protein expression.

\section{Kinome profiles accurately define tumor biopsies}

Cumulatively, our data show that measurement of kinases by MIBs capture allows integration of a significant fraction of the expressed kinome, defining a taxonomy of breast cancer determined by the functional behavior of protein kinases. This kinome taxonomy is used below to define a subset of understudied and well characterized kinases that are capable of distinguishing between breast cancer tumor subtypes.

Given both the variation in kinase MIB-binding profiles observed across subtypes, as well as their differing information content when compared to RNA expression measurements, we sought to better understand which kinases were key nodes in the subtype-selective baseline breast cancer kinome. To address this question, we investigated the MIB-binding behavior of kinases across all four subtypes. We considered three major classes of kinases: 1) those that show variation in MIB-binding across all subtypes, 2) those that exhibit more limited subtype-specific behaviors, and 3) kinases that have nominal distinguishing behavior. Standard application of PCA identifies those kinases displaying the greatest variation across all samples ("pan-subtype kinases"; Figure 2E) and thus we used a feature selection approach based on the Bhattacharyya distance [23] to determine subtype-specific kinases that are highly distinguishing/ informative for a single cancer subtype (see Methods). Integrated with PCA-identified kinases ("pan-subtype"), this combined set of the 50 most informative kinases is shown in Figure 4A, with column ordering based on similarity of the kinome profile and recapitulating similarity between claudin-low and basal-like subtypes as well as HER2-enriched and luminal. The HER2+ cell line that profiles as basal-like (HCC1954, in purple) is displayed in its own column. Recognized cancer-related kinases are again observed in this set, including ERBB2, FGFR2, PTK6, RAF1 and RON (MST1R) as well as 22 understudied kinases.

The kinases shown in Figure 4A have the greatest variation within and across subtypes and are representative of each of the major subfamilies of kinases in addition to three metabolic kinases captured by MIBs (Figure 4B). Under the assumption that TNBC (represented by the basal-like and claudin-low cell lines) and HER2/luminal breast cancer are separate diseases, we again used unsupervised feature selection of MIB/MS data to identify kinases that distinguish TNBC (basal-like/claudin-low) from HER2/luminal breast cancer [23]. As shown in Figure $4 \mathrm{C}$, obvious differences in the kinome profiles of TNBC and HER2/luminal are observed, demonstrating the unique functional phenotypic features of the kinome in the two different breast cancers. Sixteen understudied kinases showed strong variance between TNBC and HER2/ luminal breast cancer, with higher-ranked understudied kinases being DAPK3, ADCK1, MRCKA (CDC42BPA), STK17A, DMPK and VRK2.

Using the kinases chosen through feature selection and shown in Figure 4C, we evaluated the ability to use MIB-binding profiles to define subtypes of human HER2+ needle biopsies and TNBC breast tumors (Figure 4D and $4 \mathrm{E}$ ). Diagnostic needle biopsies of 2 patient tumors (2 HER2+) having $\sim 1 \mathrm{mg}$ of total protein were processed using MIB enrichment. With just $1 \mathrm{mg}$ of tumor lysate protein, the total number of kinases purified from each biopsy ranged from $\sim 200$ to 275 . Utilizing only the kinases identified from cell lines as the identifying features (Figure 4C) within a SVM classifier, it was possible to clearly identify HER2+ and TNBC primary patient tumors. We note that heterogeneity within these tumor samples was significant, with between 15 and 27 of our 50 most informative kinases not being measured/ measurable within these primary samples, supporting the need for the identification of a larger number of kinases showing dynamic behavior across subtypes. Of further interest, we found that markers such as ERBB2 were not required for accurate classification of HER2+ cancers i.e., exclusion of ERBB2 as a feature during classification led to the same classifications of primary tumors shown in Figure 4D and 4E. These results indicate that subtype information was encoded across multiple other kinases as assessed through MIB/MS. Thus, MIB-binding activity of significantly less than 50 kinases is sufficient to discriminate the functional phenotypic nature of the kinome in breast cancer. As with cell lines, application of 

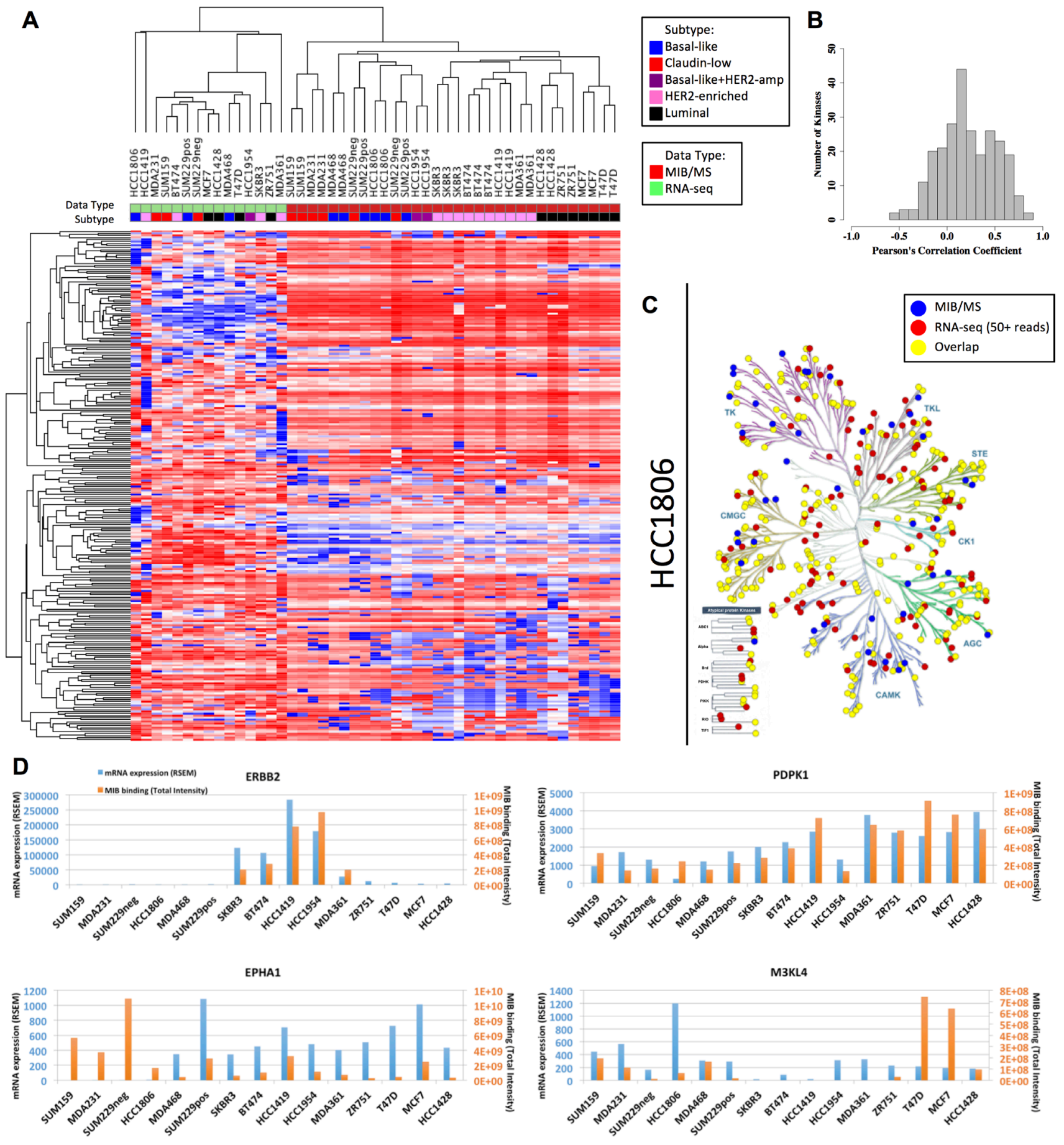

Figure 3: Overall MIB-binding and mRNA expression levels are not correlated. (A) Heatmap of autoscaled mRNA expression and MIB-binding for all of the samples analyzed. Rows and columns are hierarchically clustered. Rows are the 254 kinases. The "Data Type" track along the columns indicates which methodology the sample is derived from (green: RNA-seq or red: MIB/MS). The "Subtype" track along the top of the heatmap indicates which subtype the cell line is classified as (blue: basal-like, red: claudin-low, purple: basallike+HER2-amp, pink: HER2-enriched, and black: luminal). (B) Frequency distribution of Pearson's correlation coefficient across all cell lines in MIB/MS and RNA-seq for each of the 254 kinases. (C) KinomeTree for MIB-binding (blue dots), RNA expression (red dots), and the overlap between the two data sets (yellow dots) for the HCC1806 (basal-like) cell line. (D) Representative raw profiles of ERBB2, PDPK1, EPHA1, and M3KL4 in MIB/MS intensity (orange bars) and mRNA RSEM counts (blue bars), showing both highly correlated and poorly correlated behavior between the two data sets. 
PCA to MIB-binding profiles showed a clear separation between TNBC tumors and HER2+ tumors (Figure 4F). Kinases driving the variation within the data included ERBB2 as might be expected, along with understudied kinases such as TESK1 and DMPK (Figure 4G).

\section{A functional interaction network of MIB-binding kinases}

To establish a basic picture of the architecture of the human kinome using MIB-bound kinases, we compiled protein interaction and phosphorylation data from multiple data sources and established a functional interaction network among 246 of the 254 kinases commonly identified in the panel of 15 breast cancer cell lines (see Methods). Spectral clustering of this network further enabled the identification of 16 subnetworks, with many showing functional enrichment of one or more Gene Ontology functional categories (Figure 5A; GO term enrichment for subnetworks is provided in Supplementary Data File 4). Of note is that understudied kinases (green nodes) are widely distributed across all the major subnetworks, demonstrating that these poorly characterized kinases are integrated into subnetworks along with well characterized kinases. The 50 distinguishing kinases identified for cell lines in Figure 4A (triangle nodes) were also distributed throughout the network and associated subnetworks. The breadth of subnetwork coverage by these kinases suggests that their predictive value in our subtype classification comes from their distribution across many subnetworks, providing an overall estimate of the state of many functional processes simultaneously.

Of the kinases bound by MIBs, we identified four Tdark kinases within our data, including ADCK1, CSK23, SG196 and M3KL4 (MAP3K21). As defined in the NIH Illuminating the Druggable Genome (IDG) program, Tdark kinases are very poorly characterized in terms of publications, small molecule inhibitors and gene references to functionality [1]. To provide an example of how MIB/MS can be used to help provide putative functional roles for such poorly characterized kinases, we utilized Lasso regression to identify potential functional linkages for these kinases as well as all understudied kinases, including those identified as Tdark and Tbio by the IDG program. As described in greater detail in Methods, known physical interactions were first identified from multiple data sources for all kinases. These were then integrated with potential functional linkages between kinases identified through Lasso regression across MIBbinding profiles. Together, these data established functional subnetworks centered on each individual understudied kinase. In total, 89 understudied kinases were annotated with such functional subnetworks, of which 18 were defined as Tbio and 4 as Tdark kinases. Functional annotation of these groups was then performed to identify enriched functional GO categories or signaling pathways, with results for all 89 kinases provided in Supplementary Data File 5.
An example of this analysis as applied to the Tdark kinase SG196 (Sugen Kinase 196 or protein-O-mannose kinase) is shown in Figure 5B. SG196 was found to have two known physical interactions in addition to eight regression linkages that reveal over-representations of the ERBB signaling pathway, MAPK cascade, and positive regulation of GTPase activity as well as several other statistically enriched biological functions as identified through GO and pathway analysis of the entire functional subnetwork identified in Figure 5B. Similarly, M3KL4 (MAP3K21) has only a single known physical interaction but by utilizing the correlated behavior of the regression kinases the functional network has overrepresentation of MAPKK activity, regulation of immune system, and response to stress (Figure 5C). The networks and functional annotations for the other Tdark kinases, CSK23 and ADCK1 are provided in Supplementary Data File 5.

\section{Kinome MIB-binding profile and response to drug perturbation}

To assess how the baseline kinome and associated understudied kinases change in their functional MIBbinding profile in response to targeted drug perturbation, we exposed four cell lines to three subtype-relevant kinase inhibitors: SUM159 and HCC1806 with trametinib (a MEK1/2 inhibitor); SKBR3 with lapatinib (a HER2/ EGFR inhibitor); and MCF7 with buparlisib (a PI3K inhibitor). Each inhibitor strongly suppressed growth of the selected subtype specific cell line (Figure 6A). We have previously shown that the kinome is dynamic and rapidly adapts to targeted perturbation by kinase inhibitors $[7,8]$. This adaptive response is readily observed by changes in the MIB-binding profiles for each drug treatment (Figure 6B, Supplementary Data File 3), with SUM159 cells showing the strongest dynamic response to drug perturbation relative to the other cell lines, but each line clearly shows an adaptive response of the kinome measured by MIB-binding profiles. Figure $6 \mathrm{C}$ shows the kinases that are unique to each subtype defined in Figure 2C (Supplementary Table 3).

Scatter plots of the SUM159 and HCC1806 dataset defines specific kinases and kinome subnetworks that drive the adaptive response to MEK1/2 inhibition that are represented by both understudied and well characterized kinases (Figure 6D). Understudied (i.e. NEK2 and PASK) and well characterized (i.e. DDR1 and EPHA4) kinases respond differently in the two subtypes (basal-like and claudin-low) when they are treated with the same kinase inhibitor (trametinib). The kinases in the subnetworks defined in Figure 5 also respond uniquely in the basal-like and claudin-low subtypes when treated with trametinib (Figure 6E). The adaptive kinome response measured by dynamic changes in MIB-binding profiles is more clearly seen when specific subnetworks are analyzed (Figure 6F). The global response of the seven largest subnetworks to 

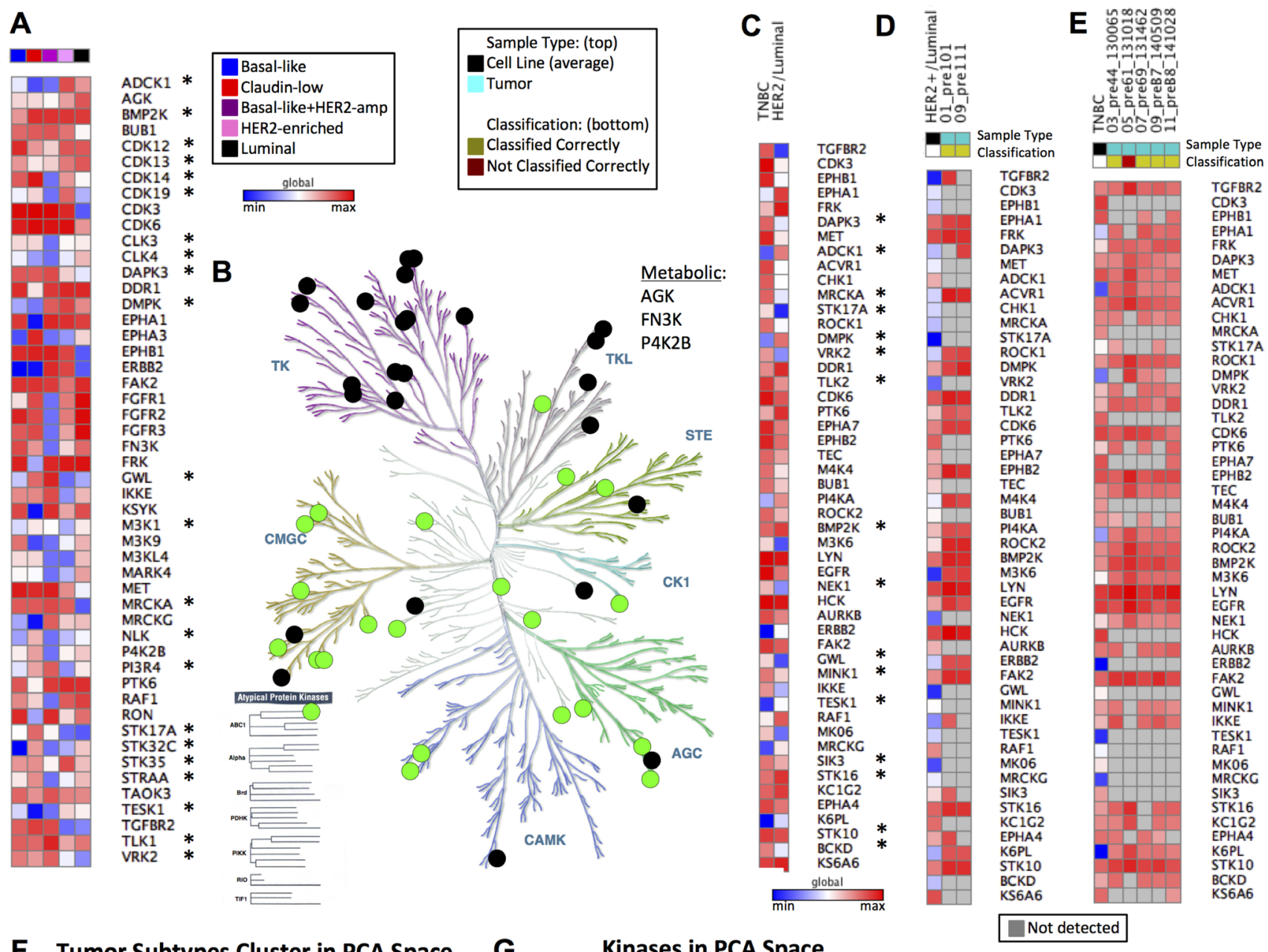

F Tumor Subtypes Cluster in PCA Space
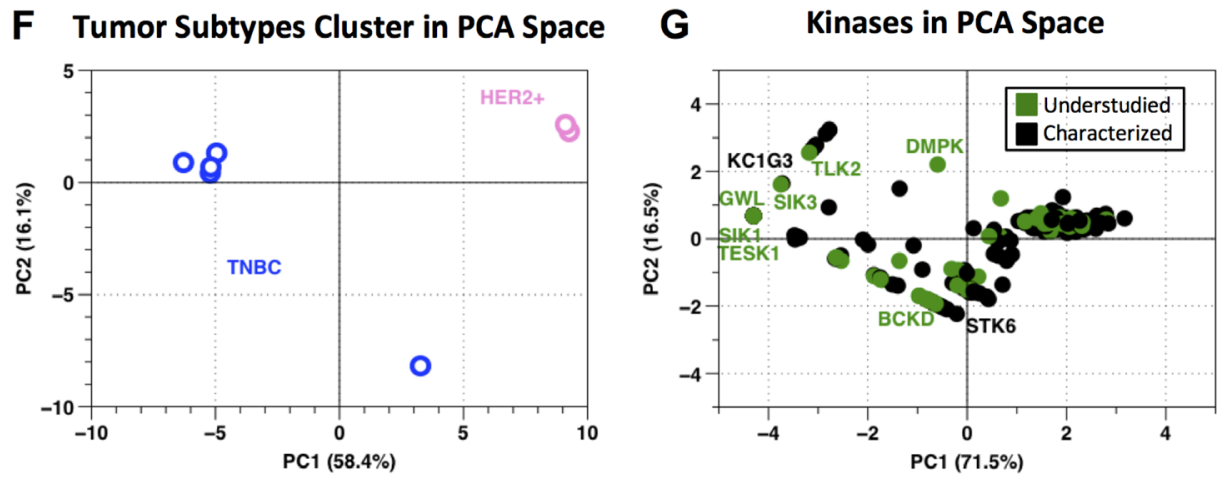

Figure 4: Baseline kinome of cell lines and tumors across breast cancer subtypes. (A) Compilation of subtype specific and pan-subtype kinases chosen from feature selection and PCA, respectively. All data is $\log 2$ normalized and autoscaled by sample, with heat map colors indicating low (blue) to high (red) MIB-binding. Column color bar indicates subtype (red: claudin-low, blue: basal-like, pink: HER2-enriched, black: luminal, purple: basal-like/HER2amp; Understudied kinases are denoted by *). Global maximum and minimum color assignment. (B) KinomeTree with the 50 distinguishing features from (A) are denoted. Black circles denote characterized kinases, while green circles represent understudied kinases. (C) Kinases chosen from feature selection when comparing Luminal/HER2-enriched cell line samples against basal-like/claudin-low (TNBC) cell line samples. Kinases are ordered from top to bottom in the same ordering as from the feature selection (most heavily weighted kinases are at the top of the heat map). All data is $\log 2$ normalized and autoscaled by sample, with heat map colors consistent with those in (A) (Understudied kinases are denoted by *). Global maximum and minimum color assignment. (D) Heat map of Luminal/HER2-enriched cell line average (HER2+/Luminal column; black in "Sample Type" column color bar) across the kinases shown in (C) with two tumor samples (teal in "Sample Type" column color bar). Data is $\log 2$ normalized and autoscaled by samples, as previously noted. Yellow in the "Classification" column bar shows which samples are classified correctly as Luminal/HER2-enriched by the SVM using the kinases from (C). Blue in the heat map indicates a low MIB-binding, red indicates high MIB-binding, and grey (in the tumor samples only) indicates that a kinase was not detected by MIBs in the tumor sample. Global maximum 
and minimum color assignment. (E) Heat map of TNBC cell line average (TNBC column; black in "Sample Type" column color bar) across the kinases shown in (C) with five tumor samples (teal in "Sample Type" column color bar). Data is log2 normalized and autoscaled by samples, as previously noted. Yellow in the "Classification" column color bar shows which samples are classified correctly as TNBC by the SVM using the kinases from (C). Dark red in "Classification" indicates that the tumor sample was incorrectly classified (not classified as TNBC) by the SVM using the kinases identified in (C). Color scheme in the heatmap is consistent with that described in (D). Global maximum and minimum color assignment. (F) PCA scores plot of tumor samples with PC1 and PC2 accounting for 58.4\% and $16.1 \%$ of variance, respectively. TNBC tumors are blue and HER2-enriched tumors are pink. (G) PCA loadings plot of tumor samples with PC1 and PC2 accounting for $71.5 \%$ and $16.5 \%$ of variance, respectively. Black points are characterized kinases and green points denote understudied kinases.

these drug perturbations is shown with their functional annotation as estimated from Gene Ontology terms and KEGG pathway enrichment shown on the $\mathrm{x}$-axis and MIB-binding response reported on the y-axis as a mean across all cell lines and drugs. Subnetworks have heterogeneous responses, with some subnetworks being fairly coordinated in response and others having kinase members acting in a more strongly divergent manner. For instance, subnetwork 3 (SN3) is enriched with many kinases relevant to cytoskeleton, adhesion and motility and has many of its members strongly up-regulated in response to drug perturbation. In comparison, SN2, involved in cell cycle and cell division, contains both strongly up- and down-regulated kinases, with the largest responses being loss of MIB-binding, consistent with the inhibition of cell growth. Understudied kinases (green labels in Figure 6F) often display large responses to drug treatment within a given subnetwork, demonstrating these kinases actively contribute to adaptive kinome reprogramming in response to targeted kinase inhibition. Similarly, a more detailed look at targeted inhibition of specific subnetworks for each of the cell lines shows the dynamic response of the kinome to be highly dependent on the drug, subtype and subnetwork context (Figure 6G, Supplementary Table 4). More broadly, the response of kinases in subnetworks is consistent with a unique functional regulation of the kinome in cancer subtypes and in response to different perturbations.

\section{DISCUSSION}

While the creation of molecular taxonomies has established the existence of subtypes in many tissuespecific cancers, how these taxonomies can be leveraged to characterize phenotype or to guide the development of targeted therapeutics remains unclear. A complication for improving therapeutic intervention with targeted kinase inhibitors in cancer is the extensive number of understudied kinases, whose poor characterization presents significant challenges to understanding their role in emergent processes such as adaptive bypass reprogramming and resistance to kinase inhibitors. Despite such challenges, understudied kinases do have potential as novel drug targets once their functional integration into signaling networks is more clearly determined. Methods generally have been lacking to capture kinases, both well characterized and understudied, to define the functional kinome en masse. Characterization of kinase MIB- binding in tumor cell lysates has proven to be a powerful technique for characterizing functional architectures of the kinome that provides the capability to identify prognostic signatures and differential response to perturbations such as targeted kinase inhibition, as well as better establishing the integration and function of understudied kinases. This is clearly seen in the 50-kinase profile distinguishing TNBC from HER2+/luminal breast cancer, with many of the 50 kinases representing understudied kinases.

The highest weighted understudied kinases distinguishing TNBC from HER2+/luminal breast cancer include ADCK1 (AarF Domain Containing Kinase whose function is unclear), DAPK3 (Death-associated protein kinase thought to be involved in apoptosis), DMPK (Dystrophia myotonica protein kinase whose function is not well-defined), MRCKA (Myotonic dystrophy kinase-related CDC42 binding protein kinase alpha that may signal CDC42 control of the actin cytoskeleton and is related to DMPK), STK17A (Serine/threonine kinase 17A has apoptosis-inducing activity and is a member of the DAP kinase-related family), TLK2 (Tousled-like kinase 2 is involved in chromatin assembly and possibly DNA repair) and VRK2 (Vaccinia-related kinase 2 that is believed to regulate apoptosis and cell growth). Screening of the cBioPortal for Cancer Genomics (http://www. cbioportal.org/public-portal/) indicates amplification of MRCKA (CDC42BPA) in 13-25\% of invasive breast cancer while TLK2 is amplified in $10 \%$ of invasive breast cancer and $25 \%$ of adenoid cystic breast cancer. ADCK1, DAPK3, DMPK STK17A and VRK2 were found to be similarly amplified in other cancers including prostate adenocarcinoma, uterine carcinosarcoma and pancreatic adenocarcinoma. Prominent MIB-binding signatures combined with potential increased expression in tumors suggests these understudied kinases have important functions for the tumor cell phenotype that have not been characterized to date.

While MIB/MS provides a unique picture of the functional state of the kinome, this approach does come with its own challenges. Binding of a kinase to an immobilized inhibitor is dependent on multiple factors including inhibitor selectivity, total protein concentration, functional activation state, non-specific binding as well as other variables. The choice of inhibitors used here was done to provide a broad picture of kinome behavior, which could potentially improve with testing of additional complementary inhibitor combinations. We also note that our analysis is based on a 

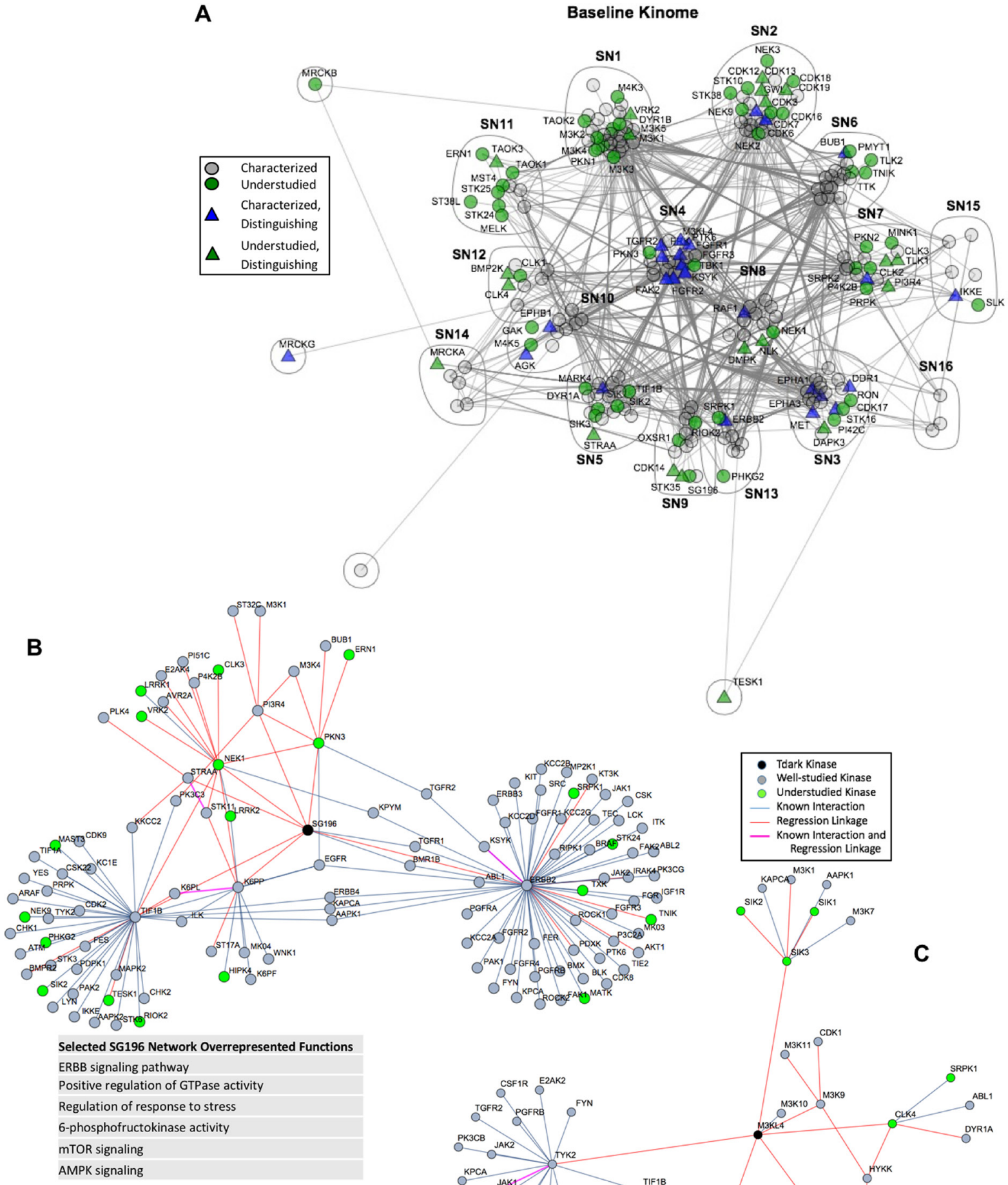

Selected M3KL4 Network Overrepresented Functions

Activation of MAPKK activity

IL-17 signaling

PI3K cascade

RAF-independent MAPK1/3 activation

IGF1R signaling cascade

Figure 5: Subnetworks in the functional kinome. (A) Compiled and spectral clustered protein-protein interaction network from public data sources of the 246 out of 254 kinases analyzed in the MIB/MS cell line data set. Green nodes represent understudied kinases, while grey and blue nodes represent well characterized kinases. Triangles are kinases that are also in the distinguishing features found in Figure 4A. (B and C) Local functional network for SG196 (B) and M3KL4 (C) as defined through Lasso regression of MIB/MS data with sample enriched annotations from GO, Kegg and Reactome pathways. 

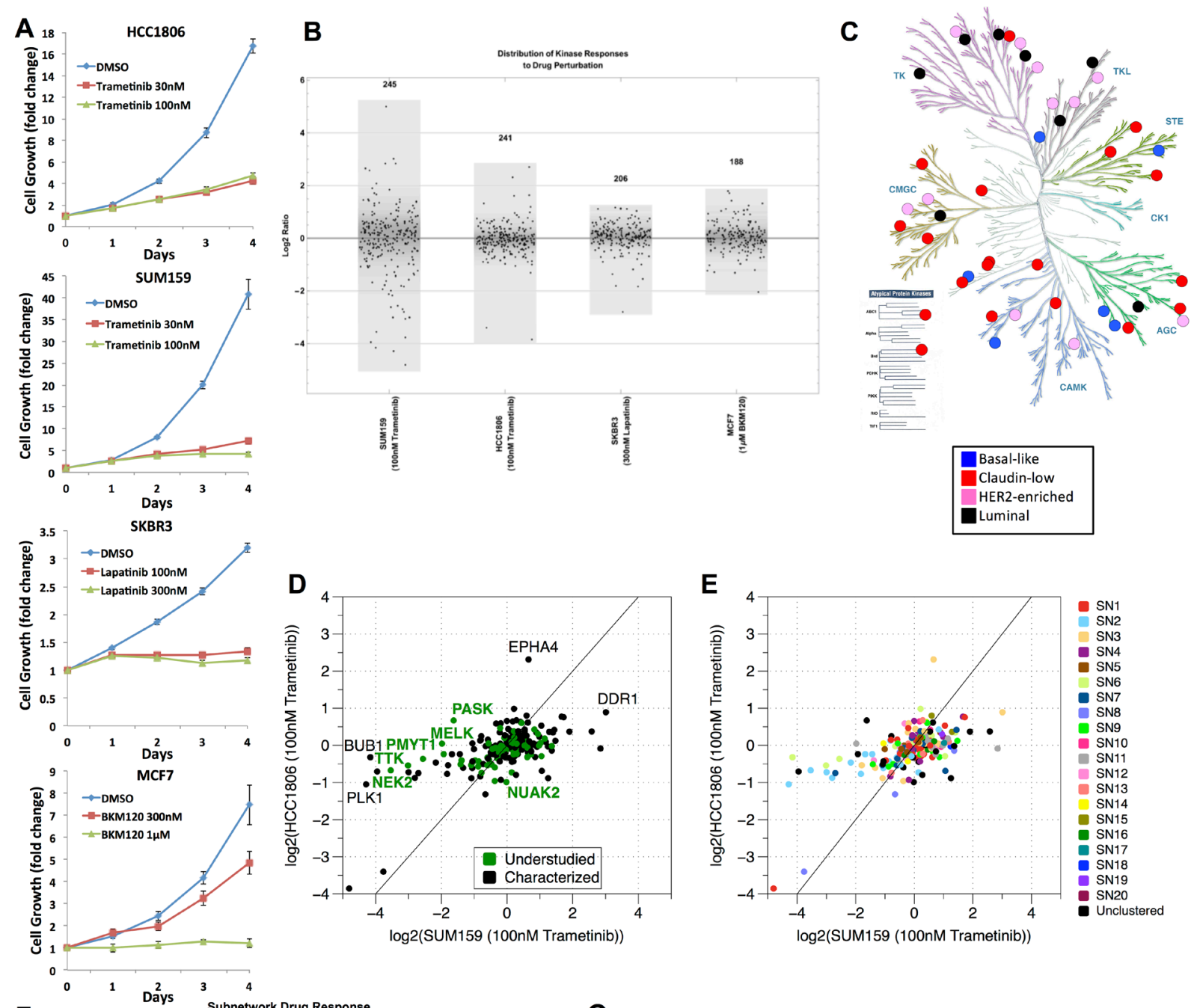

$\mathbf{F}_{3}$
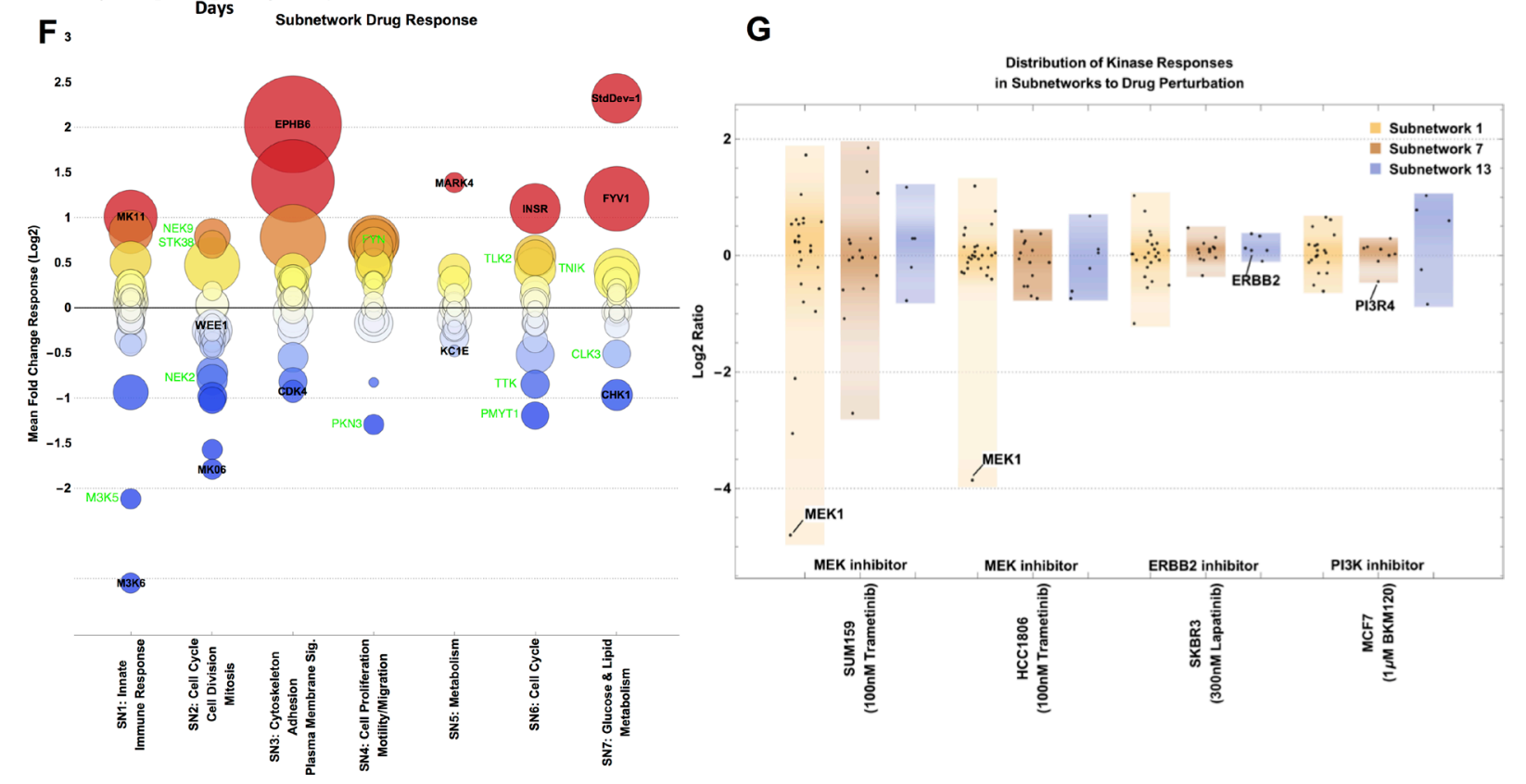

Figure 6: Kinome drug response overall and by subnetwork. (A) Growth curves for HCC1806 + Trametinib, SUM159 + Trametinib, SKBR3 + Lapatinib, and MCF7 + Buparlisib (i.e. BKM120). All curves were done at two different doses. (B) Response of kinome in representative cell lines across four subtypes of breast cancer (claudin-low: SUM159, basal-like: HCC1806, HER2-enriched: SKBR3, luminal: MCF7) when treated with the indicated kinase inhibitor. Distribution of the kinome response on the log2-scale is shown 
for each cell line/subtype; each point represents a kinase. (C) KinomeTree showing the kinases that are uniquely captured in each of the subtypes in the baseline data set. Blue circles denote kinases bound to the MIBs only in basal-like samples. Similarly, red circles represent claudin-low, pink circles represent HER2-enriched, and black circles represent luminal uniquely bound kinases. (D) Scatter plot of the response of the basal-like vs. claudin-low cell lines to treatment with $100 \mathrm{nM}$ Trametinib. All values are fold change to untreated cells and $\log 2$-transformed. Kinase points are colored black for characterized and green for understudied. (E) Scatter plot of the response of the basal-like vs. claudin-low cell lines to treatment with $100 \mathrm{nM}$ Trametinib (same as in (D)). Kinases are colored by subnetwork assigned to each kinase from Figure 5. (F) Subnetwork response to drug perturbation showing mean fold change across the four representative cell lines (SUM159, HCC1806, SKBR3, and MCF7) for the top 7 subnetworks identified from Figure 5. Characterized and understudied kinases in each subnetwork are labeled in black and green, respectively. The color of each circle indicates the mean fold change (red $=$ high/above 0 , blue $=$ low/below 0 ), while the area of the circle denotes the standard deviation of the fold changes across the representative cell lines. $(\mathbf{G})$ Distribution of the kinome response in the three subnetworks SN1, SN7, and SN13 on the log2-scale is show for each cell line/subtype.

sampling of 15 breast cancer cell lines and we expect that other informative kinases will be identified, and some of our identified kinases down-weighted, as additional samples are acquired. Molecular heterogeneity inherent within primary tumors will require the characterization of greater samples, though it is encouraging that kinases identified through cell lines were able to make relatively reliable classifications. The complementary nature of MIB-binding profiles with those derived from gene expression would suggest that combining profiles from these data types would likely improve future classification approaches as well as enhance understanding of regulatory relationships. Regardless, these results support the potentially significant role of post-translational and posttranscriptional regulation in kinome dynamics that drives differences between these two measures of kinome behavior.

The dynamic nature of the kinome is clearly captured in the kinase MIB-binding profiles characterizing baseline versus post-drug treated cells. This adaptive reprogramming of the kinome is involved in the epigenetic development of resistance to kinase inhibitors [24]. We have proposed that blocking this adaptive reprogramming is important clinically for making single kinase inhibitors more durable [8]. Pre- and post-drug treatment MIB/MS analysis allows for the quantitative measure of kinome adaptive responses and the rapid screening of combinations of kinase or epigenetic inhibitors that would block the adaptive behavior of the kinome $[7,8,24]$. This analysis can be done in preclinical models as well as patient trials where biopsy accessible tumor specimens are available. We have been able to capture more than 200 kinases with as little as 300 micrograms (ug) of protein. Thus, MIBs provide a proteomic approach to characterize the functional state and dynamics of the kinome and thus define therapeutic response and targetable adaptive resistance networks. Importantly, MIBs capture both well characterized and understudied kinases for a comprehensive measure of the functional kinome.

\section{MATERIALS AND METHODS}

\section{MIB affinity chromatography}

Broad spectrum Type I kinase inhibitors (CTx0294885, VI-16832, PP58, Purvalanol B, UNC-2147A, and UNC-8088A) were custom-synthesized with hydrocarbon linkers and terminal amine groups and covalently attached to ECH-activated Sepharose beads as previously described [8]. Cells were rinsed in PBS and processed in lysis buffer (50 mM HEPES, $150 \mathrm{mM}$ $\mathrm{NaCl}, 0.5 \%$ Triton X-100, 1 mM EDTA, 1 mM EGTA, at $\mathrm{pH} 7.5$ containing $10 \mathrm{mM} \mathrm{NaF}, 2.5 \mathrm{mM} \mathrm{NaVO}$, cOmplete protease Inhibitor Cocktail (Roche), and 1\% Phosphatase Inhibitor Cocktails 2 and 3 (Sigma)). Tumor biopsies obtained from UNC Tissue Procurement were manually homogenized with a chilled mortar and pestle in lysis buffer. For individual bead profiling (Figure 1), $2 \mathrm{mg}$ of total protein was gravity-flowed over $100 \mathrm{uL}$ of each bead. For Figure 2 (cell lines), $5 \mathrm{mg}$ of total protein lysate and for Figure 4 (human tumor biopsies), $1 \mathrm{mg}$ of total protein was gravity-flowed over a mixture of the six kinase inhibitor-linked beads (175 uL total beads). Beads were washed with at least 30 volumes of high salt $(1 \mathrm{M}$ $\mathrm{NaCl})$ and low salt $(150 \mathrm{mM} \mathrm{NaCl})$ lysis buffer, then 500 $\mathrm{uL}$ of low salt lysis buffer containing $0.1 \%$ SDS. Bound proteins were eluted by boiling with $0.5 \%$ SDS and $1 \%$ $\beta$-mercaptoethanol in $100 \mathrm{mM}$ Tris- $\mathrm{HCl}, \mathrm{pH} 6.8,2 \mathrm{X}$ 15 minutes, treated with DTT $\left(5 \mathrm{mM}, 25 \mathrm{~min}\right.$ at $\left.60^{\circ} \mathrm{C}\right)$ and Iodoacetamide ( $20 \mathrm{mM}, 30 \mathrm{~min}$ in the dark at RT), and spin-concentrated to $100 \mu \mathrm{L}$ (Millipore Amicon Ultra-4, $10 \mathrm{~K}$ cutoff) before Methanol/Chloroform precipitation. Proteins were trypsinized overnight at $37^{\circ} \mathrm{C}$ and then dried down in a speed-vac. Peptides were cleaned with C-18 spin columns (Pierce).

\section{Mass spectrometry and analysis}

Peptides were resuspended in $2 \% \mathrm{ACN}$ and $0.1 \%$ Formic Acid. For Figure 1 (bead profiling) 20\% of each sample was injected onto a Thermo Easy-Spray $75 \mu \mathrm{m} \times$ $15 \mathrm{~cm} \mathrm{C}-18$ column using an Easy nLC-1000 in technical triplicate and separated on a 150 min gradient $(5-40 \%$ $\mathrm{ACN}$ ). For Figures 2 and 4 (cell lines and tumor biopsies), $40 \%$ of the final peptide suspension was injected onto an Easy-Spray $75 \mu \mathrm{m} \times 25 \mathrm{~cm} \mathrm{C}-18$ column and separated on a 300 min gradient (cell lines) or a 180 min gradient (tumor biopsies). For all runs, ESI parameters: 3e6 AGC MS1, 80 ms MS1 max inject time, 1e5 AGC MS2, 100 ms MS2 max inject time, 20 loop count, $1.8 \mathrm{~m} / \mathrm{z}$ isolation window, 
$45 \mathrm{~s}$ dynamic exclusion. Spectra were searched against the Uniprot/Swiss-Prot database with Sequest HT on Proteome Discoverer software (Figures 1 and 2) or MaxQuant (Figure 4). Only peptides with medium or greater confidence (5\% FDR) were considered for quantitation, and only kinases having 3 or more unique peptides were considered for further analysis. Heat maps were generated using GENE-E software (BROAD institute). Kinome trees were generated using Kinome Render (http://bcb.med. usherbrooke.ca/kinomerender.php).

\section{RNA-seq}

Total RNA was spin column purified using RNeasy Plus Mini kit (Qiagen). Library construction was performed at the UNC Lineberger Comprehensive Cancer Center Genomics Core and the sequencing at the UNC High-Throughput Sequencing Facility. mRNA-Seq libraries were constructed with $1 \mu \mathrm{g}$ total RNA using the Illumina TruSeqTMRNA Sample Prep Kit according to the manufacturer's protocol. 50-cycled single-end sequencing runs with multiplexing were produced using an Illumina HiSeq2000. CASAVA 1.8.2 generated bases and assessed sequence quality. The QC-passed reads were aligned to the hg19 human reference genome using MapSplice and the alignment profile was determined by Picard Tools v1.64 [25]. Aligned reads were sorted and indexed using SAMtools, and then translated to transcriptome coordinates and filtered for indels, large inserts, and zero mapping quality using UBU v1.0. Transcript abundance estimates for each sample were determined using an Expectation-Maximization algorithm [26]. Publicly available data from $[27,28]$ were also processed using this computational method. Data is available in Supplementary Data File 2.

\section{Data analysis}

The MIB/MS data set was filtered so that only kinases that are represented in at least $30 \%$ of the 32 samples were analyzed. Initially, over 380 kinases were in the MIB/MS data set but 254 kinases passed this filtering threshold. The MIB/MS data set was $\log 2$ transformed and mean-centered, variance-scaled by sample before downstream analysis. Hierarchical clustering, Principal Components Analysis (PCA), and feature selection were performed in MATLAB. PCA is a commonly-used data analysis and dimension-reduction technique that transforms variables into a set of linearly uncorrelated principal components [29]. Application of PCA also provides the ability to assign a weight to each feature (kinase) in the data set that can be used as a relative measure of its ability to distinguish subtypes. To identify kinases that dominate individual PCs, kinases having weights in the 90th-percentile (i.e. those weighted in the top $10 \%$ of weights) per PC were selected from the first three PCs and used in downstream classification tasks. Feature selection using the Bhattacharyya distance was also used as a secondary mechanism for ranking kinases in terms of their ability to distinguish subtypes [23]. Pairwise classification between subtypes (e.g. basal-like subtype from all others, claudin-low from all others, etc) was iteratively performed to identify the most informative features for each subtype.

Kinases identified through feature ranking and PCA are combined to create a list of the most distinguishing kinases in MIB-binding across the breast cancer subtypes. Subtype-specific signature kinases are defined as the top $5 \%$ of the highest-ranking kinases found using the Bhattacharyya feature ranking coefficient for each subtype are compiled for the overall list. Pan-subtype kinases are defined as the most heavily weighted kinases (top 10\%) from the first three PCs are used. Subtype-specific kinases are compiled from each of the breast cancer subtypes then the pan-subtype kinases are added (in order from most heavily weighted to less heavily weighted) starting with PC1 kinases then moving to PC2 then to PC3 until a maximum of 50 kinases is reached to make up the list of distinguishing kinases.

\section{Comparison of MIB-binding to transcript abundance}

The Z-score is calculated by sample based on the average $\log 2$ value per kinase and using the standard deviation of all kinases for a given sample for both data types, MIB/MS and RNA-seq (Figure 3A). The Pearson correlation of individual kinases is calculated for each kinase across the 15 cell lines (not distinguished by subtype) between MIB-binding and RNA transcript levels.

\section{Prediction of subtypes}

Classification of subtype based on a previously unobserved kinome profile was performed using a Support Vector Machine (SVM). The SVM is a machine learning technique used in supervised classification, and thus requires a training set on which to learn parameters that can then be applied towards prediction of previously unobserved data [30]. The SVM used here utilized the 50 distinguishing kinases previously identified in cell lines to predict the subtype of human primary tumors. Human tumors are classified into one of the major groups (TNBC or HER2+/Luminal) or as "other".

\section{Network analysis}

Protein-protein interaction information was compiled from multiple public data sources for the 254 kinases analyzed in this data set and included, the Human Integrated Protein-Protein Interaction rEference (HIPPIE) (updated 9/1/2015; [31]), Human Protein Reference 
Database (HPRD Release 9; [32]), Interlogous Interaction Database (I2D version 2.9; [33, 34]), PhosphoSitePlus (phosphosite.org - downloaded 10/15/2015; [35]) and Reactome protein-protein interactions (downloaded $12 / 15 / 2015$; [36]). Out of the 254 kinases in the MIB data set, 246 were found to have kinase to kinase interactions in the public data sets. The union of all interactions between the 246 kinases was used to form a single network that was then clustered into communities/subnetworks with the spectral method in Mathematica (ver 10.3). Subnetworks were assessed for GO-term enrichment via Panther [37].

\section{Annotation of Tdark kinases}

To identify sets of kinases that were functionally linked through common MIB-binding behavior, we utilized Lasso regression, which has strong utility as a feature selection tool [38]. Lasso regression was performed on each of the 254 kinases which passed initial filtering (from the Supplementary Data File 1). Iterating through all kinases, a single kinase's MIB/MS data was used as the response vector while all other kinases formed the input matrix. The regression was performed in $\mathrm{R}$ utilizing the glmnet package [39]. The resulting features for each Tdark kinase, and the kinases which had it as a resulting feature, are all labeled as regression correlations in Figure 5. Known interactions from earlier described public data sources were identified for each Tdark kinase and each of its regression correlations. The final Tdark kinase networks were created from the combination of both regression correlations and known interactions. All kinases involved in each Tdark kinase network were listed and compared against a list of all human kinases to find overrepresented GO biological processes, Kegg and Reactome pathways via Panther [37] and g:Profiler [40].

\section{Data and materials availability}

All processed data is provided in Supplementary Data Files. Data from the Sequence Read Archive is used for some analyses here: SRX317702, SRX317711, SRX317712, SRX317715, SRX317717, SRX317723, SRX317730, SRX317733, SRX317736, SRX317738PT1, SRX317738PT2, SRX317741, SRX317743, SRX317747.

\section{Author contributions}

KALC did computational analysis, generated figures, writing, and editing. TS generated experimental (MIB/MS) data, created figures, writing, and editing. JSZ generated experimental (RNA-seq) data, writing, and editing. MPE did writing and editing. TP generated experimental (MIB/MS) data. CRH generated growth curves, functional annotation, writing, and editing. DRG generated experimental (MIB/MS) data, writing, and editing. SMB generated experimental (MIB/MS) data, writing, and editing. SPA generated experimental (MIB/MS) data, writing, and editing. SHV generated experimental (MIB/MS) data, writing, and editing. NS analyzed RNA-seq data in pipeline, writing, and editing. TIO contributed to functional annotation. LMG was involved in experimental design. GLJ managed and designed experimental work, writing, and editing. SMG managed and designed computational analyses, writing, and editing.

\section{CONFLICTS OF INTEREST}

The authors do not have any competing interests.

\section{FUNDING}

MH104999, U24DK116204 (GLJ, SMG), CA058223 (GLJ), CA177993 (SMG), GM101141 (GLJ, LMG), Susan G. Komen Foundation IIR2-225201 (GLJ), GM116534 (SMB), Komen Foundation PDF15331014 (SHV), CA189205 and CA118100 (TIO), T32 CA009156 (TJS), UNC Junior Faculty Development Award (JSZ), University Research Council Small Grant Award (JSZ), University Cancer Research Fund (GLJ).

\section{REFERENCES}

1. Nguyen DT, Mathias S, Bologa C, Brunak S, Fernandez N, Gaulton A, Hersey A, Holmes J, Jensen LJ, Karlsson A, Liu G, Ma'ayan A, Mandava G, et al. Pharos: collating protein information to shed light on the druggable genome. Nucleic Acids Res. 2017; 45:D995-1002. https://doi.org/10.1093/nar/ gkw1072.

2. Santos R, Ursu O, Gaulton A, Bento AP, Donadi RS, Bologa CG, Karlsson A, Al-Lazikani B, Hersey A, Oprea TI, Overington JP. A comprehensive map of molecular drug targets. Nat Rev Drug Discov. 2017; 16:19-34. https://doi. org/10.1038/nrd.2016.230.

3. Fedorov O, Müller S, Knapp S. The (un)targeted cancer kinome. Nat Chem Biol. 2010; 6:166-69. https://doi. org/10.1038/nchembio.297.

4. Cancer Genome Atlas Network. Comprehensive molecular portraits of human breast tumours. Nature. 2012; 490:61-70. https://doi.org/10.1038/nature11412.

5. Prat A, Adamo B, Fan C, Peg V, Vidal M, Galván P, Vivancos A, Nuciforo P, Palmer HG, Dawood S, Rodón J, Ramon y Cajal SR, Del Campo JM, et al. Genomic analyses across six cancer types identify basal-like breast cancer as a unique molecular entity. Sci Rep. 2013; 3:3544. https://doi. org/10.1038/srep03544. Erratum in: Sci Rep. 2015; 5:8179. Ramony Cajal, Santiago [corrected to Ramon y Cajal, Santiago].

6. Hoadley KA, Yau C, Wolf DM, Cherniack AD, Tamborero D, Ng S, Leiserson MD, Niu B, McLellan MD, Uzunangelov 
V, Zhang J, Kandoth C, Akbani R, et al, and Cancer Genome Atlas Research Network. Multiplatform analysis of 12 cancer types reveals molecular classification within and across tissues of origin. Cell. 2014; 158:929-44. https://doi.org/10.1016/j. cell.2014.06.049.

7. Duncan JS, Whittle MC, Nakamura K, Abell AN, Midland AA, Zawistowski JS, Johnson NL, Granger DA, Jordan NV, Darr DB, Usary J, Kuan PF, Smalley DM, et al. Dynamic reprogramming of the kinome in response to targeted MEK inhibition in triple-negative breast cancer. Cell. 2012; 149:307-21.

8. Stuhlmiller TJ, Miller SM, Zawistowski JS, Nakamura K, Beltran AS, Duncan JS, Angus SP, Collins KA, Granger DA, Reuther RA, Graves LM, Gomez SM, Kuan PF, et al. Inhibition of Lapatinib-Induced Kinome Reprogramming in ERBB2-Positive Breast Cancer by Targeting BET Family Bromodomains. Cell Reports. 2015; 11:390-404. https://doi. org/10.1016/j.celrep.2015.03.037.

9. Midland AA, Whittle MC, Duncan JS, Abell AN, Nakamura K, Zawistowski JS, Carey LA, Earp HS 3rd, Graves LM, Gomez SM, Johnson GL. Defining the expressed breast cancer kinome. Cell Res. 2012; 22:620-23. https://doi.org/10.1038/ cr.2012.25.

10. Daub H, Olsen JV, Bairlein M, Gnad F, Oppermann FS, Körner R, Greff Z, Kéri G, Stemmann O, Mann M. Kinase-selective enrichment enables quantitative phosphoproteomics of the kinome across the cell cycle. Mol Cell. 2008; 31:438-48. https://doi.org/10.1016/j.molcel.2008.07.007.

11. Delmore JE, Issa GC, Lemieux ME, Rahl PB, Shi J, Jacobs HM, Kastritis E, Gilpatrick T, Paranal RM, Qi J, Chesi M, Schinzel AC, McKeown MR, et al. BET bromodomain inhibition as a therapeutic strategy to target c-Myc. Cell. 2011; 146:904-17. https://doi.org/10.1016/j.cell.2011.08.017.

12. Marcotte R, Brown KR, Suarez F, Sayad A, Karamboulas K, Krzyzanowski PM, Sircoulomb F, Medrano M, Fedyshyn Y, Koh JL, van Dyk D, Fedyshyn B, Luhova M, et al. Essential gene profiles in breast, pancreatic, and ovarian cancer cells. Cancer Discov. 2012; 2:172-89. https://doi.org/10.1158/21598290.CD-11-0224.

13. Jordan NV, Prat A, Abell AN, Zawistowski JS, Sciaky N, Karginova OA, Zhou B, Golitz BT, Perou CM, Johnson GL. SWI/SNF chromatin-remodeling factor Smarcd3/Baf60c controls epithelial-mesenchymal transition by inducing Wnt5a signaling. Mol Cell Biol. 2013; 33:3011-25. https://doi. org/10.1128/MCB.01443-12.

14. Prat A, Cheang MC, Martín M, Parker JS, Carrasco E, Caballero R, Tyldesley S, Gelmon K, Bernard PS, Nielsen TO, Perou CM. Prognostic significance of progesterone receptorpositive tumor cells within immunohistochemically defined luminal A breast cancer. J Clin Oncol. 2013; 31:203-09. https://doi.org/10.1200/JCO.2012.43.4134.

15. Hedenfalk I, Duggan D, Chen Y, Radmacher M, Bittner M, Simon R, Meltzer P, Gusterson B, Esteller M, Kallioniemi OP, Wilfond B, Borg A, Trent J, et al. Gene-expression profiles in hereditary breast cancer. N Engl J Med. 2001; 344:539-48. https://doi.org/10.1056/NEJM200102223440801.

16. Sotiriou C, Neo SY, McShane LM, Korn EL, Long PM, Jazaeri A, Martiat P, Fox SB, Harris AL, Liu ET. Breast cancer classification and prognosis based on gene expression profiles from a population-based study. Proc Natl Acad Sci USA. 2003; 100:10393-98. https://doi.org/10.1073/pnas.1732912100.

17. Zhang B, Wang J, Wang X, Zhu J, Liu Q, Shi Z, Chambers MC, Zimmerman LJ, Shaddox KF, Kim S, Davies SR, Wang S, Wang P, et al, and NCI CPTAC. Proteogenomic characterization of human colon and rectal cancer. Nature. 2014; 513:382-87. https://doi.org/10.1038/nature13438.

18. Franks A, Airoldi E, Slavov N. Post-transcriptional regulation across human tissues. PLoS Comput Biol. 2017; 13:e1005535. https://doi.org/10.1371/journal.pcbi.1005535.

19. Sendoel A, Dunn JG, Rodriguez EH, Naik S, Gomez NC, Hurwitz B, Levorse J, Dill BD, Schramek D, Molina H, Weissman JS, Fuchs E. Translation from unconventional 5' start sites drives tumour initiation. Nature. 2017; 541:494-99. https://doi.org/10.1038/nature21036.

20. de Sousa Abreu R, Penalva LO, Marcotte EM, Vogel C. Global signatures of protein and mRNA expression levels. Mol Biosyst. 2009; 5:1512-26.

21. Karaman MW, Herrgard S, Treiber DK, Gallant P, Atteridge CE, Campbell BT, Chan KW, Ciceri P, Davis MI, Edeen PT, Faraoni R, Floyd M, Hunt JP, et al. A quantitative analysis of kinase inhibitor selectivity. Nat Biotechnol. 2008; 26:127-32. https://doi.org/10.1038/nbt1358.

22. Davis MI, Hunt JP, Herrgard S, Ciceri P, Wodicka LM, Pallares G, Hocker M, Treiber DK, Zarrinkar PP. Comprehensive analysis of kinase inhibitor selectivity. Nat Biotechnol. 2011; 29:1046-51. https://doi.org/10.1038/nbt.1990.

23. Bhattacharyya A. On a Measure of Divergence between Two Statistical Populations Defined by Their Probability Distributions. Bull Calcutta Math Soc. 1943; 35:99-109.

24. Zawistowski JS, Bevill SM, Goulet DR, Stuhlmiller TJ, Beltran AS, Olivares-Quintero JF, Singh D, Sciaky N, Parker JS, Rashid NU, Chen X, Duncan JS, Whittle MC, et al. Enhancer Remodeling during Adaptive Bypass to MEK Inhibition Is Attenuated by Pharmacologic Targeting of the P-TEFb Complex. Cancer Discov. 2017; 7:302-21. https:// doi.org/10.1158/2159-8290.CD-16-0653.

25. Wang K, Singh D, Zeng Z, Coleman SJ, Huang Y, Savich GL, He X, Mieczkowski P, Grimm SA, Perou CM, MacLeod JN, Chiang DY, Prins JF, et al. MapSplice: accurate mapping of RNA-seq reads for splice junction discovery. Nucleic Acids Res. 2010; 38:e178. https://doi.org/10.1093/nar/gkq622.

26. Li B, Dewey CN. RSEM: accurate transcript quantification from RNA-Seq data with or without a reference genome. BMC Bioinformatics. 2011; 12:323. https://doi. org/10.1186/1471-2105-12-323.

27. Daemen A, Griffith OL, Heiser LM, Wang NJ, Enache OM, Sanborn Z, Pepin F, Durinck S, Korkola JE, Griffith M, Hur 
JS, Huh N, Chung J, et al. Modeling precision treatment of breast cancer. Genome Biol. 2013; 14:R110. https://doi. org/10.1186/gb-2013-14-10-r110. Erratum in: Genome Biol. 2015; 16:95.

28. Heiser LM, Sadanandam A, Kuo WL, Benz SC, Goldstein TC, Ng S, Gibb WJ, Wang NJ, Ziyad S, Tong F, Bayani N, $\mathrm{Hu}$ Z, Billig JI, et al. Subtype and pathway specific responses to anticancer compounds in breast cancer. Proc Natl Acad Sci USA. 2012; 109:2724-29. https://doi.org/10.1073/ pnas. 1018854108 .

29. Slack MD, Martinez ED, Wu LF, Altschuler SJ. Characterizing heterogeneous cellular responses to perturbations. Proc Natl Acad Sci USA. 2008; 105:19306-11. https://doi.org/10.1073/ pnas.0807038105.

30. Furey TS, Cristianini N, Duffy N, Bednarski DW, Schummer M, Haussler D. Support vector machine classification and validation of cancer tissue samples using microarray expression data. Bioinformatics. 2000; 16:906-14. https://doi. org/10.1093/bioinformatics/16.10.906.

31. Schaefer MH, Fontaine JF, Vinayagam A, Porras P, Wanker EE, Andrade-Navarro MA. HIPPIE: Integrating protein interaction networks with experiment based quality scores. PLoS One. 2012; 7:e31826. https://doi.org/10.1371/journal. pone.0031826.

32. Keshava Prasad TS, Goel R, Kandasamy K, Keerthikumar S, Kumar S, Mathivanan S, Telikicherla D, Raju R, Shafreen B, Venugopal A, Balakrishnan L, Marimuthu A, Banerjee S, et al. Human Protein Reference Database - 2009 update. Nucleic Acids Res. 2009; 37:D767-72. https://doi.org/10.1093/nar/ gkn892.
33. Brown KR, Jurisica I. Online predicted human interaction database. Bioinformatics. 2005; 21:2076-82. https://doi. org/10.1093/bioinformatics/bti273.

34. Brown KR, Jurisica I. Unequal evolutionary conservation of human protein interactions in interologous networks. Genome Biol. 2007; 8:R95. https://doi.org/10.1186/gb-2007-8-5-r95.

35. Hornbeck PV, Zhang B, Murray B, Kornhauser JM, Latham V, Skrzypek E. PhosphoSitePlus, 2014: mutations, PTMs and recalibrations. Nucleic Acids Res. 2015; 43:D512-20. https:// doi.org/10.1093/nar/gku1267.

36. Croft D, Mundo AF, Haw R, Milacic M, Weiser J, Wu G, Caudy M, Garapati P, Gillespie M, Kamdar MR, Jassal B, Jupe S, Matthews L, et al. The Reactome pathway knowledgebase. Nucleic Acids Res. 2014; 42:D472-77. https://doi.org/10.1093/ nar/gkt1102.

37. Mi H, Huang X, Muruganujan A, Tang H, Mills C, Kang D, Thomas PD. PANTHER version 11: expanded annotation data from Gene Ontology and Reactome pathways, and data analysis tool enhancements. Nucleic Acids Res. 2017; 45:D183-89. https://doi.org/10.1093/nar/gkw1138.

38. Tibshirani R. Regression Shrinkage and Selection Via the Lasso. J R Stat Soc Series B Stat Methodol. 1994; 58:267-88.

39. Friedman J, Hastie T, Tibshirani R. Regularization Paths for Generalized Linear Models via Coordinate Descent. J Stat Softw. 2010; 33:1-22. https://doi.org/10.18637/jss.v033.i01.

40. Reimand J, Arak T, Adler P, Kolberg L, Reisberg S, Peterson H, Vilo J. g:Profiler-a web server for functional interpretation of gene lists (2016 update). Nucleic Acids Res. 2016; 44:W839. https://doi.org/10.1093/nar/gkw199. 